\title{
Three-dimensional structure of clumpy outflow from supercritical accretion flow onto black holes
}

\author{
Hiroshi KOBAYASHI, ${ }^{* 1,2}$ Ken OHSUGA, ${ }^{2,1}$ Hiroyuki R. TAKAHASHI, ${ }^{3}$ \\ Tomohisa KAWASHIMA, ${ }^{2}$ Yuta ASAHINA, ${ }^{3}$ Shun TAKEUCHI, ${ }^{4}$ and Shin \\ MINESHIGE ${ }^{5}$
}

${ }^{1}$ School of Physical Sciences, Graduate University of Advanced Study (SOKENDAI), Shonan Village, Hayama Kanagawa 240-0193

${ }^{2}$ Division of Theoretical Astronomy, National Astronomical Observatory of Japan, 2-21-1

Osawa,Mitaka-shi, Tokyo 181-8588

${ }^{3}$ Center for Computational Astrophysics, National Astronomical Observatory of Japan, 2-21-1 Osawa,Mitaka-shi, Tokyo 181-8588

44-1-10-103, Okusawa, Setagaya, Tokyo 158-0083

${ }^{5}$ Department of Astronomy, Graduate School of Science, Kyoto University, Kitashirakawa, Oiwake-cho, Sakyo-ku, Kyoto 606-8502

*E-mail: hiro.kobayashi@nao.ac.jp

Received ; Accepted

\begin{abstract}
We perform global three-dimensional (3D) radiation-hydrodynamic (RHD) simulations of outflow from supercritical accretion flow around a $10 M_{\odot}$ black hole. We only solve the outflow part, starting from the axisymmetric 2D simulation data in a nearly steady state but with small perturbations in a sinusoidal form being added in the azimuthal direction. The mass accretion rate onto the black hole is $\sim 10^{2} L_{\mathrm{E}} / c^{2}$ in the underlying $2 \mathrm{D}$ simulation data and the outflow rate is $\sim 10 L_{\mathrm{E}} / c^{2}$ (with $L_{\mathrm{E}}$ and $c$ being the Eddington luminosity and speed of light, respectively). We first confirm the emergence of clumpy outflow, which was discovered by the 2D RHD simulations, above the photosphere located at a few hundreds of Schwarzschild radii $\left(r_{\mathrm{S}}\right)$ from the central black hole. As prominent 3D features we find that the clumps have the shape of a torn sheet, rather than a cut string, and that they are rotating around the central black hole with a sub-Keplerian velocity at a distance of $\sim 10^{3} r_{\mathrm{S}}$ from the center. The typical clump size is $\sim 30$ $r_{\mathrm{S}}$ or less in the radial direction, and is more elongated in the angular directions, $\sim$ hundreds of $r_{\mathrm{S}}$ at most. The sheet separation ranges from 50 to $150 r_{\mathrm{S}}$. We expect stochastic time variations when clumps pass across the line of the sight of a distant observer. Variation timescales are estimated to be several seconds for a black hole with mass of ten to several tens of $M_{\odot}$, in rough agreement with the observations of some ultra-luminous $\mathrm{X}$-ray sources.
\end{abstract}

Key words: accretion, accretion disks — black hole physics — hydrodynamics — instabilities — radiation: dynamics 


\section{Introduction}

The significant and unique roles of supercritical (or superEddington) accretion flow in astrophysical objects have been recognized quite recently. The most remarkable features of supercritical accretion flow are intense high-energy radiation and massive outflow (see Chapter 10 of Kato, Fukue, \& Mineshige 2008 and references therein), both of which inevitably have a great impact on its environment (King 2003; Ohsuga et al. 2005; Ohsuga \& Mineshige 2011; Sa̧dowski et al. 2014).

Supercritical accretion is now thought to occur in a variety of objects. Objects powered by supercritical accretion are sometimes called as "super-Eddington accretors". Good examples (or candidates) are ultraluminous X-ray sources (ULXs; Fabbiano et al. 1989; Watarai et al. 2001; King et al. 2001; Swartz et al. 2004) and ultraluminous supersoft X-ray sources (ULSs; Di Stefano \& Kong 2003; Urquhart \& Soria 2016, Gu et al. 2016; Ogawa et al. 2017). In addition, some microquasars (Watarai \& Mineshige 2003; Done et al. 2007; Pakull et al. 2010; Vierdayanti et al. 2010) and some of the narrowline Seyfert 1 galaxies (e.g., Wang et al. 1999; Mineshige et al. 2000) may fall onto this category. More recently, ULX pulsars and neutron-star systems have joined the group of superEddington accretors (Bachetti et al. 2014; Fürst et al. 2016; Kawashima et al. 2016; Israel et al. 2017; Takahashi \& Ohsuga 2017; Takahashi et al. 2017). It has also been suggested that supermassive black holes might have experienced supercritical accretion phase in their formation epoch (see e.g., Inayoshi et al. 2016 and references therein).

Generally speaking, emergence of outflow seems to be ubiquitous in any accretion system. In particular, powerful outflows from various types of black hole objects have now been observationally established through a number of observations ; see, Halpern (1984), Reynolds (1997), Kaastra et al. (2000), and Tombesi et al. (2013) for AGN outflow, and Boirin et al. (2004), Church et al. (2005), Ueda et al. (2009), and Miller et al. (2015) for outflow from black hole binaries. Given this, it should be interesting to study how they affect their environments. Interaction between supermassive black holes and their environments, so-called AGN feedback, is now being studied from various viewpoints in relation to possible co-evolution of supermassive black holes and their host galaxies (e.g. Silk \& Rees 1998; Kormendy \& Ho 2013). Since the energy output in either form of radiation or outflow from super-Eddington accretors is enormous, the feedback effects should be even more important for super-Eddington accretors. In fact, ULXs are occasionally accompanied by ULX nebulae, ionized regions surrounding them, although their physical origins are still an open question at the moment (see, e.g., Feng \& Soria 2011 and references therein). It might be useful to note here that the enormous impacts of super-Eddington objects, other than black hole objects, have also been extensively discussed in various astrophys- ical contexts, including luminous blue variables (LBVs), WolfRayet stars, classical novae, supernovae and so on (Davidson \& Humphreys 1997; Nugis \& Lamers 2000; Shaviv 2000; Smith et al. 2009).

Global multi-dimensional, radiation-hydrodynamics (RHD) /radiation-magnetohydrodynamics (radiation-MHD) simulations of super-Eddington accretors are being carried out rather extensively by many groups (e.g., Eggum et al. 1988; Okuda \& Fujita 2000; Ohsuga et al. 2005; 2009; Sạdowski et al. 2014; McKinney et al. 2014; Fragile et al. 2014; Jiang et al. 2014; Hashizume et al. 2015; Takahashi et al. 2016). Intense radiation from supercritical accretion flows is shown to drive outflow, by which significant amounts of mass, momentum, and energy of gas can be blown away (Fukue 2004; Takeuchi et al. 2009; Krumholz \& Thompson 2012), but the nature of the gas outflow is not well understood yet. By contrast, the outgoing radiation part has been rather intensively discussed in relation to observations. The most characteristic features inherent to superEddington accretors are found in hard X-ray ranges at around $\sim 10 \mathrm{keV}$ (e.g., Gladstone et al. 2009). There is a broad spectral bump, which can be understood in terms of Compton upscattering of soft photons within radiation-pressure-driven outflow (e.g., Kawashima et al. 2009, 2012; Narayan et al. 2017; Kitaki et al. 2017).

Here, we address one question: how is matter blown away? Part of the reason for the poor understanding of the outflow resides in the need for large-box simulations with good spatial resolution for clarifying the outflow properties. (In the sub-Eddington regime, by contrast, observable intense radiation mainly originates from the black hole vicinity so that large simulation boxes are not always necessary.) Takeuchi, Ohsuga, and Mineshige (2013, hereafter T13) were the first to perform largebox RHD simulations, and made a very important discovery in this context: they found the emergence of clumpy outflow from photosphere of super-Eddington accretors. The typical size of the clumps (clouds), $\sim 10 r_{\mathrm{S}}$, corresponds to about one optical depth, with $r_{\mathrm{S}}=2 G M_{\mathrm{BH}} / c^{2}$ being the Schwarzschild radius, where $G$ is the gravitational constant, $M_{\mathrm{BH}}$ is the mass of the black hole, and $c$ the speed of light. This fact implies that a sort of radiation hydrodynamic instability is involved with this clump formation, in addition to the well-known Rayleigh-Taylor instability in the radiation-pressure-dominated atmosphere (see also Takeuchi et al. 2014, hereafter T14). We wish to stress that the presence of clumpy features was also observationally indicated through the significant time variabilities that could be due to porous outflow from luminous objects (Fabrika 2004; Middleton et al. 2011; Tombesi et al. 2012). It might be that the BLR clouds originate from clumpy outflow (T13; see similar suggestions by Nicastro 2000; Elitzur \& Ho 2009; Elitzur 2012).

There are a few remarks on the previous simulations (T13). 
First, large simulation boxes are essential to find clumpy outflow, since it is expected to appear above a photosphere located at a few hundreds of $r_{\mathrm{S}}$ from a black hole. Second, magnetic fields do not play a principal role in clump formation, since purely RHD simulations can also produce clumpy outflow (T13; T14). [A photon-bubble instability (Arons 1992; Turner et al. 2005) is not a primary cause of clump formation.] We thus do not need to incorporate MHD processes. Finally, the previous $2 \mathrm{D}$ study was restricted to the axisymmetric approximation. Hence, neither the three-dimensional (3D) clump shape nor turbulent motion accompanying non-axisymmetric flow, if any, could be investigated there. It is even unclear if clumpy outflow does appear in 3D simulations. Then, a next question will be: what are the 3D shapes of outflow clumps?

In the present study we thus aim to clarify the $3 \mathrm{D}$ nature of the clumpy outflow by performing global, 3D RHD simulations of supercritical outflow. We will show rather unique 3D features of clumpy outflow that were not anticipated by the $2 \mathrm{D}$ simulations. The plan of this paper is as follows: In the next section we describe our model, basic equations and assumptions, and numerical procedures. We then show our results on 3D outflow properties in section 3 . Comparison with the $2 \mathrm{D}$ cases is also examined there. The final section is devoted to a discussion on the observational implications.

\section{Model and calculation methods}

\subsection{Basic equations}

In the present study, we solve the full set of RHD equations that take the terms up to the order of $(v / c)$ :

$\frac{\partial \rho}{\partial t}+\nabla \cdot(\rho \boldsymbol{v})=0$

is the continuity equation,

$\frac{\partial}{\partial t}(\rho \boldsymbol{v})+\boldsymbol{\nabla} \cdot\left(\rho \boldsymbol{v} \boldsymbol{v}+p_{\mathrm{gas}} \boldsymbol{I}\right)=-\rho \boldsymbol{\nabla} \psi_{\mathrm{PN}}-\boldsymbol{S}_{1}$,

is the momentum equation of gas,

$\frac{\partial E_{\text {gas }}}{\partial t}+\nabla \cdot\left(E_{\text {gas }}+p_{\text {gas }}\right) \boldsymbol{v}=-\rho \boldsymbol{v} \cdot \nabla \psi_{\mathrm{PN}}-c S_{0}$,

is the energy equation of gas,

$\frac{1}{c^{2}} \frac{\partial \boldsymbol{F}_{\mathrm{rad}}}{\partial t}+\nabla \cdot \mathbf{P}_{\mathrm{rad}}=\boldsymbol{S}_{1}$

is the momentum equation of radiation, and

$\frac{\partial E_{\mathrm{rad}}}{\partial t}+\boldsymbol{\nabla} \cdot \boldsymbol{F}_{\mathrm{rad}}=c S_{0}$,

is the energy equation of radiation; the source terms in the momentum and energy equations are explicitly written as

$$
\begin{aligned}
\boldsymbol{S}_{1} & =\rho \kappa_{\mathrm{ff}} \frac{\boldsymbol{v}}{c}\left(\frac{4 \pi B}{c}-E_{\mathrm{rad}}\right) \\
& -\rho\left(\kappa_{\mathrm{ff}}+\kappa_{\mathrm{es}}\right) \frac{1}{c}\left[\boldsymbol{F}_{\mathrm{rad}}-\left(\boldsymbol{v} E_{\mathrm{rad}}+\boldsymbol{v} \cdot \mathbf{P}_{\mathrm{rad}}\right)\right],
\end{aligned}
$$

and

$$
\begin{aligned}
S_{0} & =\rho \kappa_{\mathrm{ff}}\left(\frac{4 \pi B}{c}-E_{\mathrm{rad}}\right) \\
& +\rho\left(\kappa_{\mathrm{ff}}-\kappa_{\mathrm{es}}\right) \frac{\boldsymbol{v}}{c^{2}} \cdot\left[\boldsymbol{F}_{\mathrm{rad}}-\left(\boldsymbol{v} E_{\mathrm{rad}}+\boldsymbol{v} \cdot \mathbf{P}_{\mathrm{rad}}\right)\right] .
\end{aligned}
$$

Here, $\rho$ is the matter density, $\boldsymbol{v}$ is the flow velocity, $E_{\text {gas }} \equiv$ $e_{\text {gas }}+\rho \boldsymbol{v}^{2} / 2$ is the total energy of the gas (with $e_{\text {gas }}$ being the internal energy density of the gas), $p_{\text {gas }}$ is the gas pressure, $B=\sigma T_{\text {gas }}^{4} / \pi$ is the blackbody intensity (with $\sigma$ and $T_{\text {gas }}$ being the Stefan-Boltzmann constant and the temperature of the gas, respectively), $\boldsymbol{I}$ is the unit matrix, $E_{\mathrm{rad}}$ is the radiation energy density, $\boldsymbol{F}_{\text {rad }}$ is the radiative flux vector, and $\mathbf{P}_{\text {rad }}$ is the radiation pressure tensor. For simplicity, we adopt the gray (frequency-integrated) approximation for the radiation terms. Neither self-gravity nor magnetic fields are taken into account. We incorporate general relativistic effects by adopting the pseudo-Newtonian potential, $\psi_{\mathrm{PN}}=-G M_{\mathrm{BH}} /\left(r-r_{\mathrm{S}}\right)$, where $r$ is the distance from the origin (Paczyńsky \& Wiita 1980). We consider the electron scattering opacity, $\kappa_{\mathrm{es}}$, and the Rosseland mean free-free absorption opacity, $\kappa_{\mathrm{ff}}$,

$\kappa_{\mathrm{es}}=\sigma_{\mathrm{T}} m_{\mathrm{p}}^{-1}$,

and

$\kappa_{\mathrm{ff}}=1.7 \times 10^{-25} \mathrm{~m}_{\mathrm{p}}^{-2} \rho T_{\mathrm{gas}}^{-7 / 2} \mathrm{~cm}^{2} \mathrm{~g}^{-1}$,

where $m_{\mathrm{p}}$ is the proton mass and $\sigma_{\mathrm{T}}$ is the Thomson scattering cross-section

The set of equations (1)-(6) can be closed by using an ideal gas equation of state,

$p_{\text {gas }}=(\gamma-1) e_{\text {gas }}=\frac{\rho k_{\mathrm{B}} T_{\text {gas }}}{\mu m_{\mathrm{p}}}$,

and by adopting the M1-closure, which gives the radiation pressure tensor as a function of the radiation energy density as well as the radiative flux (Levermore 1984). Here, $\gamma=5 / 3$ is the specific-heat ratio, $k_{\mathrm{B}}$ is the Boltzmann constant, and $\mu=0.5$ is the mean molecular weight. Throughout the present study, we employ $M_{\mathrm{BH}}=10 M_{\odot}$.

\subsection{Numerical procedures}

In the present study we use cylindrical coordinates $(R, \theta, z)$, where $R$ is the radial distance from the rotation $(z-)$ axis, $\theta$ is the azimuthal angle, and $z$ is the vertical coordinate. We first performed a 2D simulation (same as T13, except for the size of the computational box) in order to produce the initial conditions for the 3D simulation. In the 2D simulation, computational domain extends from the vicinity of the black hole $\left(\sim 2 r_{\mathrm{S}}\right)$ to the outflow region of $10^{3} r_{\mathrm{S}}$. A quasi-steady disk, of which the mass accretion rate onto the black hole $\left(\dot{M}_{\text {acc }}\right)$ is $\simeq 10^{2} L_{\mathrm{E}} / c^{2}$ and the radiation luminosity is $\sim L_{\mathrm{E}}$, forms in a few sec after the start of the simulation, where $L_{\mathrm{E}}$ is the Eddington luminosity. We adopt part of the 2D data at $5 \mathrm{~s}$, but with perturbations being added afterwards (see below) as the initial condition of the 


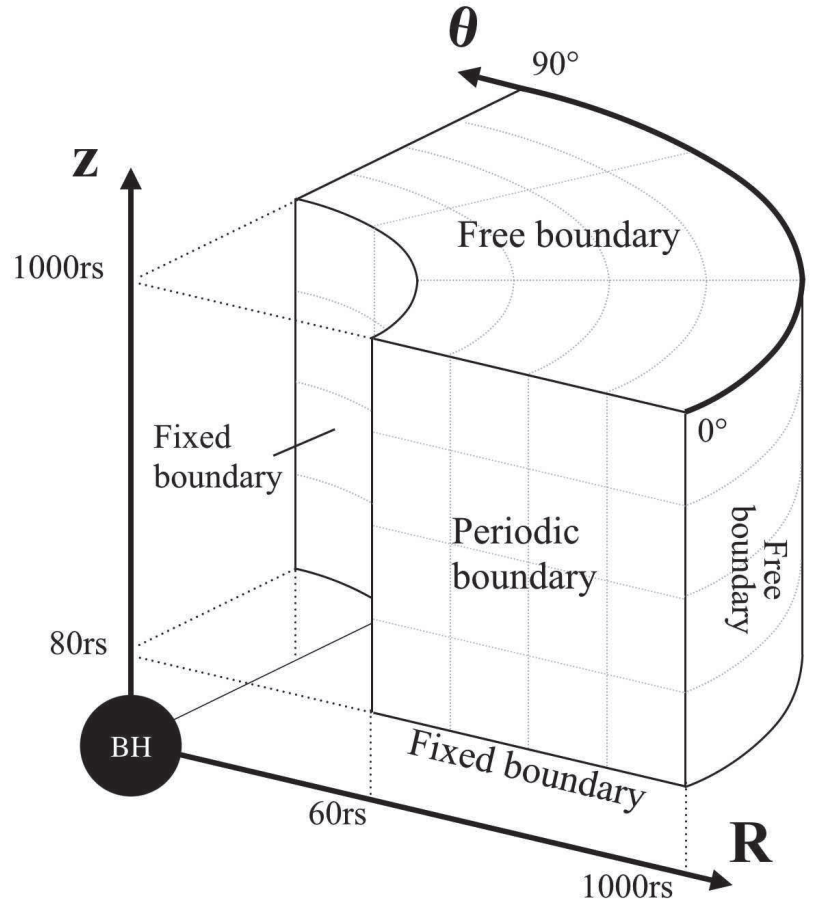

Fig. 1. Density contours used as the initial condition for the 3D simulations (see text).

3D simulation. To clarify the initial state, we show the crosssectional view of the initial density distribution on the $R$ - $z$ plane in Figure 1. We have confirmed that the 3D results do not appreciably alter, even if we adopt the $2 \mathrm{D}$ data at a different time ( $8 \mathrm{sec}$, for instance) as the initial data for the 3D simulation.

Since the original 2D data obtained above is axisymmetric $(\partial / \partial \theta=0)$, we provide small perturbations as follows:

$q(R, \theta, z)=q_{0}(R, z) \times[1+0.10 \sin (4 \theta)]$,

where $q_{0}$ represents the physical quantities of the original 2D data and $q$ is the initial values used in the present 3D simulations. We confirm that our results do not appreciably change, even if we employ random numbers between -1 and 1 instead of $\sin (4 \theta)$ at each grid point.

The computational domain of the 3D simulation is restricted to $60 \leq R / r_{\mathrm{S}} \leq 10^{3}, 0 \leq \theta \leq 90^{\circ}$, and $80 \leq z / r_{\mathrm{S}}<10^{3}$, to reduce the numerical cost. At the inner boundary at $R=60 r_{\mathrm{S}}$ or at the lower boundary at $z=80 r_{\mathrm{S}}$ we keep the same values for the physical quantities (such as matter density, temperature, velocity vectors, radiation energy density, and radiative flux) at all later times in the 3D simulation. That is, we employ fixed boundary conditions at the inner and lower boundaries. This implies that a steady disk with $\dot{M}_{\text {acc }} \sim 10^{2} L_{\mathrm{E}} / c^{2}$ is postulated to exist just inside and below the simulation box throughout the $3 \mathrm{D}$ simulation. We adopt free boundary conditions at the outer and upper boundaries $\left(R=10^{3} r_{\mathrm{S}}\right.$ and $\left.z=10^{3} r_{\mathrm{S}}\right)$. If the $R$-component ( $z$-component) of the velocity is negative, it

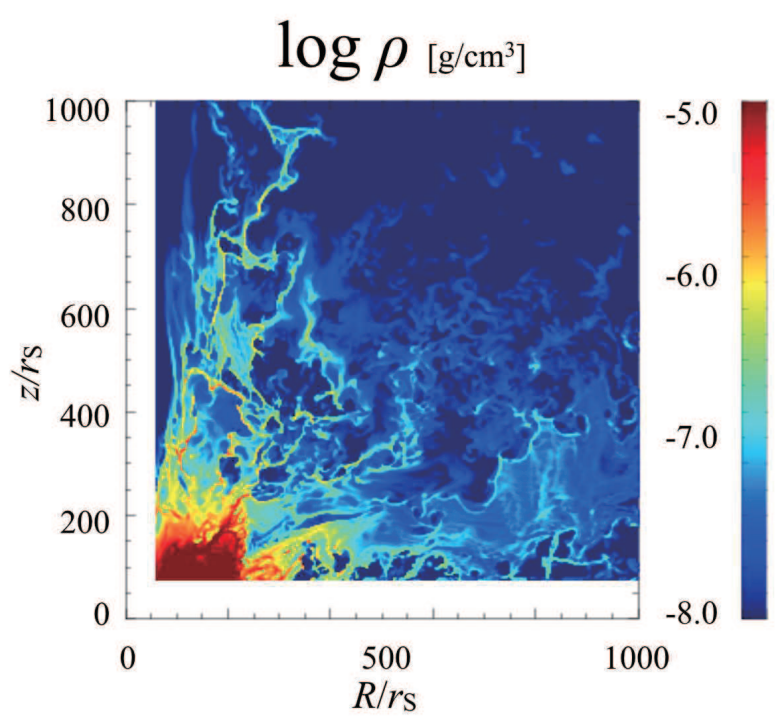

Fig. 2. Geometry of the calculation box and the adopted boundary conditions.

is automatically set to be zero at the outer (upper) boundary. Thus, matter can go out freely but not enter through the boundaries. The periodic boundary condition is used in the $\theta$-direction so that the mass, momentum, energy, and radiation across the boundary at $\theta=0^{\circ}$ are the same as those across the boundary at $\theta=90^{\circ}$. The simulation box and adopted boundary conditions are summarized in Figure 2. The grid spacing is $\Delta R=\Delta z=4.0$ $r_{\mathrm{S}}$ and $\Delta \theta=0.9^{\circ}$, respectively. Here we note that the numerical resolution somewhat affects the size of clumps (discussed later).

Our total simulation time is $12 \mathrm{~s}$, which is much longer than the wind crossing time; $10^{3} r_{\mathrm{S}} / v_{r} \sim 1.0 \mathrm{~s}$ for the black hole mass of $10 M_{\odot}$ and the radial wind velocity of $v_{r} \sim 0.1 c$. We thus conclude that we finally achieve a quasi-steady structure of the clumpy outflow.

For comparison purposes, we also perform 2D axisymmetric simulations of which the computational domain and the grid spacing are the same as those of the 3D simulations $(60 \leq$ $R / r_{\mathrm{S}} \leq 10^{3}, 80 \leq z / r_{\mathrm{S}}<10^{3}$, and $\left.\Delta R=\Delta z=4.0 r_{\mathrm{S}}\right)$. The physical quantities of the original $2 \mathrm{D}$ data, $q_{0}$, are employed as the initial values. As is the case with the $3 \mathrm{D}$ simulations, we use free boundary conditions at the outer and upper boundaries, and fixed boundary conditions at the inner and lower boundaries.

Our numerical code is an extension of CANS+ (Matsumoto et al. 2016), which is a high-resolution MHD simulation code package developed by Chiba University. (The magnetic fields are set to be zero in our present simulations). Hydrodynamic terms are solved by CANS+, and the method for solving radiation terms is basically same as Takahashi \& Ohsuga (2013). Here we note that, while they solve fully special relativistic equations, we treat the RHD equations to $\mathcal{O}(v / c)$ in the present 
simulations.

\section{Properties of Clumpy Outflows}

\subsection{Overall structure}

We first show the overall 3D structure of the outflow gas in Figure 3. This figure displays a bird's-eye view of the matter density distribution for the regions with density being higher than $5 \times 10^{-7} \mathrm{~g} \mathrm{~cm}^{-3}$. The elapsed time is $2.5 \mathrm{~s}$ after the start of our 3D simulation (i.e., in a relatively early phase). We see multiple sheet-like structures in the outflow region (above $z \sim 400$ $r_{\mathrm{S}}$ ). If we have a closer look at each sheet, we notice that its shape is neither an ellipsoid nor a thin string (like spaghetti or linguine), but is more like a flattened string or a torn sheet (like cut fettuccine or lasagna). This feature has been made clear for the first time by the current 3D simulation, and was not anticipated by the $2 \mathrm{D}$ simulations.

Figure 4 shows a set of 2D, cross-sectional views of the spatial distribution of matter density (left panels), temperature ratio (middle), and velocity fields (right) of the 2D and 3D simulation results at the elapsed time of $5.0 \mathrm{~s}$. From the top to the bottom panels we plot the structure on the $R-z$ plane calculated by the $2 \mathrm{D}$ axisymmetric simulations, that on the $R-z$ plane (or constant $\theta$ plane) by the 3D simulation, and that on the $X-Y$ plane (constant $z$ plane), respectively. Here, we define the Cartesian coordinates as $(X, Y) \equiv(R \cos \theta, R \sin \theta)$ for a fixed height, $z$.

It is curious to see if there are qualitative differences between the $2 \mathrm{D}$ and $3 \mathrm{D}$ results. It is obvious that the $2 \mathrm{D}$ simulations miss the non-axisymmetric structure, but how about the cases seen in the $R-z$ plane?

Let us first focus on the matter density contours (left three panels). We recognize a clumpy density structure above a certain height $\left(z>\right.$ several hundreds of $\left.r_{\mathrm{S}}\right)$ not only in the $R-z$ plane but also in the $R-\theta$ planes. It is striking to note that the density contours on the $R-z$ planes are amazingly similar among $2 \mathrm{D}$ and $3 \mathrm{D}$ simulations. This justifies the $2 \mathrm{D}$ simulation results. However, the width of each clump looks somewhat thinner in the 3D calculations. This point will be quantified later based on autocorrelation function (ACF) analysis. Further, the clumpy structure tends to be weakened in the time-averaged density contours (not shown, though), meaning that the clumpy structure is not fixed but is fluctuating a lot. We will show in the next subsection that the clumpy feature is smoothed by taking the azimuthal average (again based on the ACF analysis).

It is interesting to examine the correlation of matter-related quantities and radiation-related ones by inspection of the left and middle panels. Apparently they look similar, except for the colors. This feature was already pointed out by T13, which claimed anti-correlation between the matter density and the ratio of gas temperature, $T_{\text {gas }}$, to the radiation temperature, $T_{\text {rad }}$. To be more precise, we notice that the clumpy structure (with higher density seen above $\sim 500 r_{\mathrm{S}}$ ) appears in the region where the temperature ratio is smaller; e.g., the yellow clump (dense region) extending from $(R, z)=\left(600 r_{\mathrm{S}}, 850 r_{\mathrm{S}}\right)$ to $\left(750 r_{\mathrm{S}}, 550\right.$ $\left.r_{\mathrm{S}}\right)$ in the middle-left panels corresponds to the blue region (where $T_{\text {gas }} \sim T_{\text {rad }}$ ) in the central one. This is consistent with the $2 \mathrm{D}$ results reported by T13 (see their Figure 1). A similar sort of anti-correlation is found in the $R-\theta$ plane (see the bottom-left and -middle panels).

Next we compare the velocity fields of the 2D and 3D models. We plot in the right panels of Figure 4 the azimuthal velocity $\left(V_{\theta}\right)$ normalized by the Keplerian orbital velocity, $V_{\mathrm{K}}$, defined as the azimuthal velocity with which matter can rotate on a circular orbit around the $z$-axis at a fixed $z$ :

$V_{\mathrm{K}} \equiv \sqrt{\frac{G M_{\mathrm{BH}} R^{2}}{r^{3}}}$,

with $r \equiv \sqrt{R^{2}+z^{2}}$. The black arrows represent the region where the gas velocity exceeds the escape velocity, while the white arrows indicate the region where the velocity is less.

We see a smoother $V_{\theta}$ distribution in the $2 \mathrm{D}$ simulations compared with that in the 3D. This is particularly true in the interface between the inflow region (with blue color); it is nearly straight in the $2 \mathrm{D}$ results (right panel), whereas it is not in the $3 \mathrm{D}$ result. This is due to significant turbulent motion arising in the interface, producing significant velocity fluctuations. In the region around the $z$-axis, a jet with super-Keplerian rotation velocity is launched. That is, the jet material tends to go outward, but such an expanding motion is not observed. This indicates that the jet material is confined by an external pressure asserted by matter outflow.

It is interesting to note from the bottom-right panel that the toroidal velocity is mostly sub-Keplerian at large $R\left(>400 r_{\mathrm{S}}\right)$. The rotating gas there is, however, going outward due not to the centrifugal force but to the outward radiation-pressure force. We understand that material is being blown away, keeping its angular momentum, since the radiation force in the $\theta$ direction is too small to change the angular momentum.

It is also important to note that significant sub-Keplerian rotation means a slow rotation speed, which in turn leads to long flux variation timescales caused by obscuration of central light by moving clumps. Simple timescale calculations made based on the assumption of Keplerian rotation could be grossly underestimated. This point will be discussed in the final section.

\subsection{Autocorrelation analysis}

To examine the statistical properties of clumps, it is useful to calculate ACFs (see T13). The ACF as a function of the radial and azimuthal interval $(\delta l=\delta R, \delta \theta)$ for example, is calculated by 


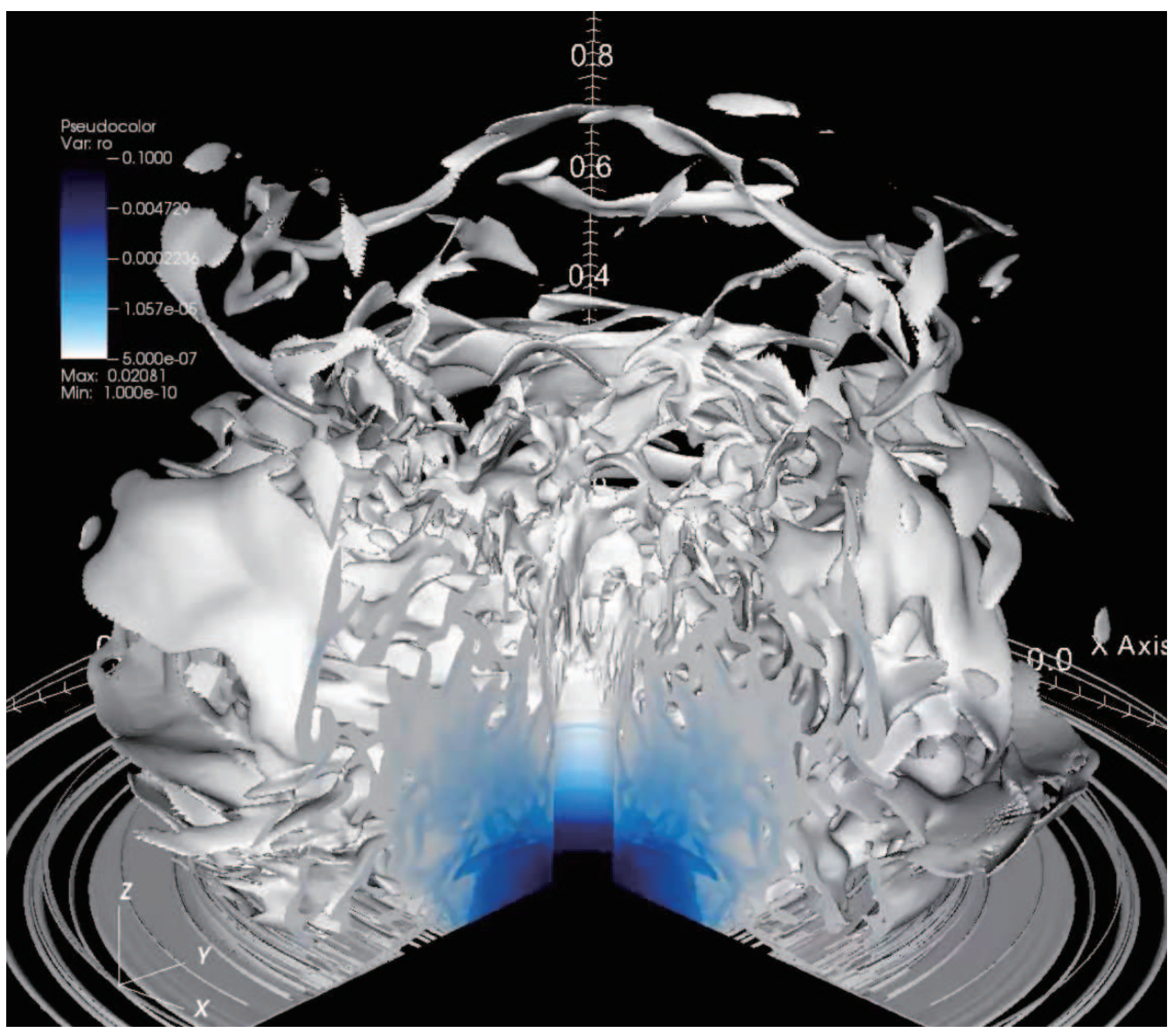

Fig. 3. Bird's eye view of the $3 \mathrm{D}$ matter density structure of an outflow from a supercritical accretion flow around a black hole with $M=10 M_{\odot}$ at the elapsed time of $t=2.5 \mathrm{~s}$. Here, we only display the regions where matter density is higher than $5 \times 10^{-7} \mathrm{~g} \mathrm{~cm}^{-3}$. The units of each axis are $10^{3} r_{\mathrm{S}}$ (i.e., the size of the calculation box). The color (silver-blue) represents matter density (see the upper-left corner for the color scale); denser regions (indicated by the blue color) are found within the inner inflow region, while less dense regions (indicated by the silver color) are mostly the outflow region. 

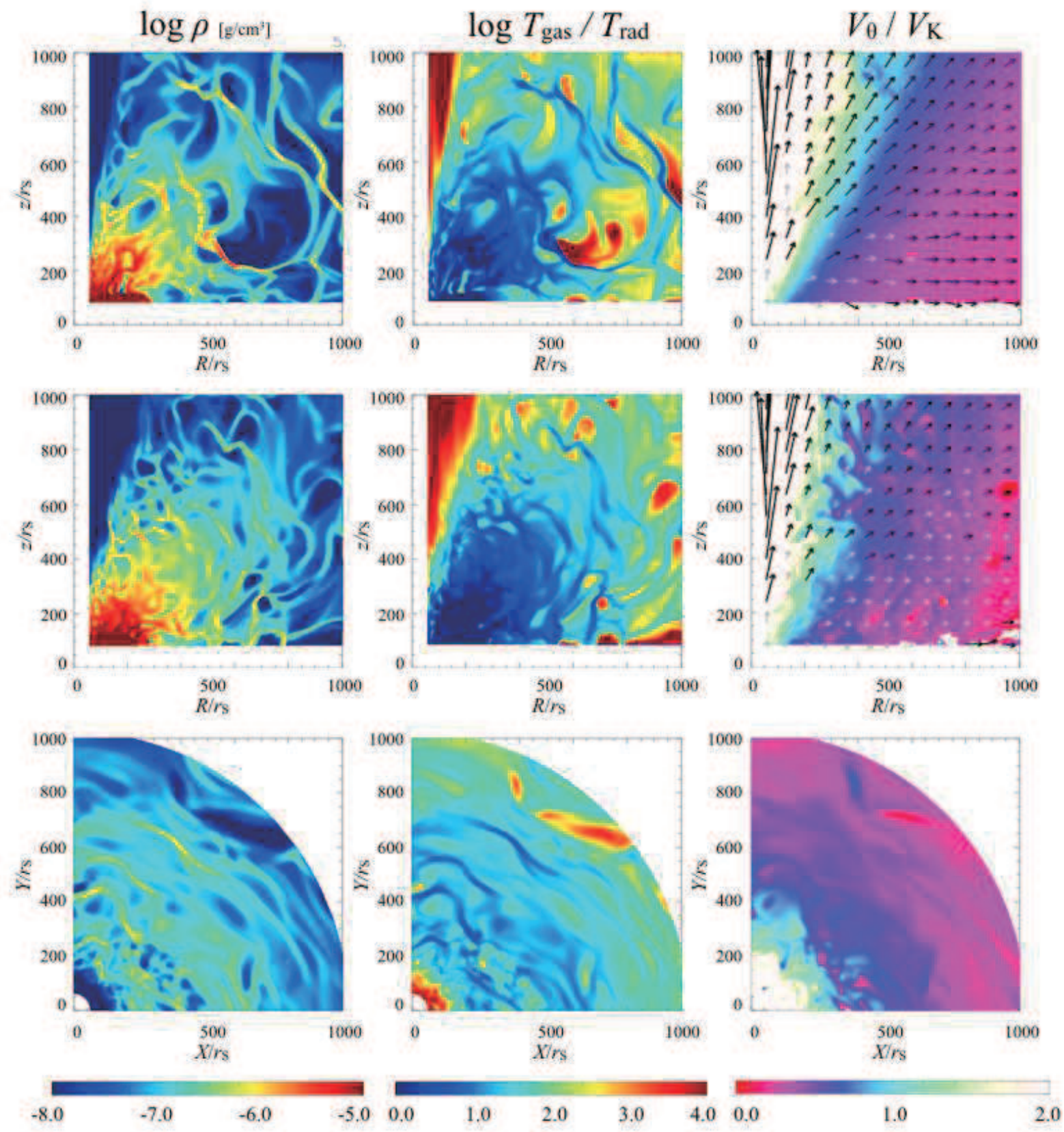

Fig. 4. Summary of the $3 \mathrm{D}$ simulation results but in two-dimensional planes in comparison with the $2 \mathrm{D}$ results. From the top to the bottom panels, we plot the cross-sectional views of the flow structure in the $R-z$ plane calculated by the 2D simulation (upper panels), those on the $R-z$ plane at $\theta=45^{\circ}$ calculated by the 3D simulations (middle panels), and those on the $X-Y$ (or $R \cos \theta-R \sin \theta$ ) plane at $z=600 r_{\mathrm{S}}$ by the 3D simulations, respectively. At each row, we plot the matter density contours (left panels), the contours of the temperature ratio $T_{\mathrm{gas}} / T_{\mathrm{rad}}$ (middle panels), and the toroidal velocity fields $V_{\theta}$ overlaid with the poloidal velocity vectors (right panels), respectively. The toroidal velocity in the right panels is normalized by the Keplerian orbital velocity, $V_{\mathrm{K}}$ (see text for the definition). Color scales are indicated below the bottom panels. The simulation parameters are the same as those in Figure 3 except for the elapsed time, which is $5 \mathrm{~s}$ here (i.e., in the quasi-steady outflow state). Note the clumpy structures seen in all the matter density contours. 


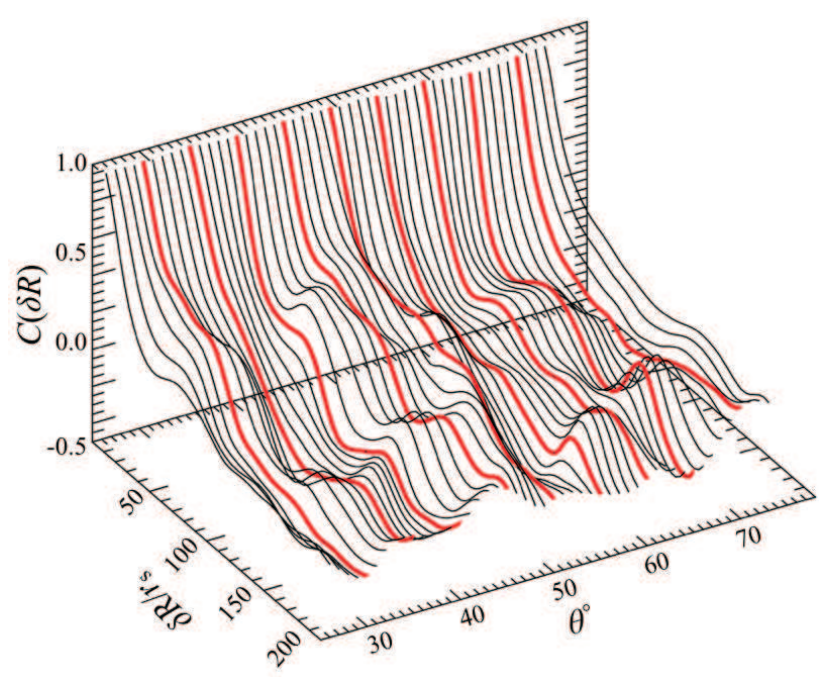

Fig. 5. Autocorrelation functions of the matter density distribution displayed in the lower-left panel in figure 4 as functions of $\delta R$ for a variety of azimuthal angles $\theta$. We colored some lines red for visual clarity.

$C(\delta l) \equiv \frac{\sum_{k=0}^{N-L-1}\left(\rho_{k+L}-\bar{\rho}\right)\left(\rho_{k}-\bar{\rho}\right)}{\sum_{k=0}^{N-1}\left(\rho_{k}-\bar{\rho}\right)^{2}}$,

where $\bar{\rho}$ is the average density,

$\bar{\rho} \equiv \frac{1}{N} \sum_{k=0}^{N-1} \rho_{k}$,

$N$ is the total number of grid points in the radial $(N=235)$ and azimuthal $(N=100)$ directions, the subscript $k$ represents the grid number, and $L$ is an integer related to $\delta l$ as $\delta R=l \times \Delta R$ and $\delta \theta=l \times \Delta \theta$, where we took a constant grid spacing : $\Delta R=$ $4.0 r_{\mathrm{S}}$ and $\Delta \theta=0.9^{\circ}$.

Figure 5 illustrates the ACFs calculated based on the matter density data on the $X-Y$ plane (shown in the middle-left panel of figure 4) as functions of $\delta R / r_{\mathrm{S}}$ at various angles of $\theta$. Here, we fix $z=600 r_{\mathrm{S}}$. See also the red line in the upper panel of Figure 6, which is the one-dimensional plot of $C(\delta R)$ at a fixed $(\theta, z)=\left(45^{\circ}, 600 r_{\mathrm{S}}\right)$.

From these ACF profiles, we can extract the typical size of clumps and typical interval between neighboring clumps in the following way: First, the width of the primary peak (at around $\delta R=0)$ represents the typical size of each clump. We find similar slopes of each ACF near $\delta R \sim 0$ in Figure 5, and hence understand that the half-width of the clump is $\ell_{\mathrm{cl}}^{r} / 2 \sim 15 r_{\mathrm{S}}$ regardless of the azimuthal angles. Second, the interval between the primary peak and the secondary one at $\delta R=50-150 r_{\mathrm{S}}$ represents the separation between the neighboring clumps. We see significant variations in the ACF shape around its second peaks, indicating different clump intervals at different $\theta$. In other words, there is no coherence in the clump distributions.
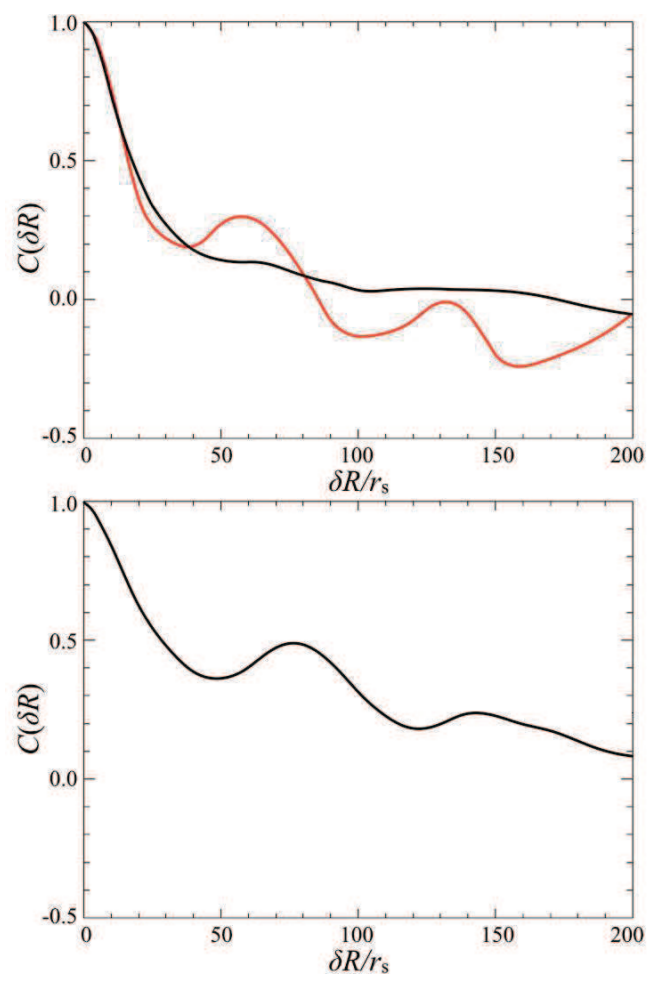

Fig. 6. Comparisons of the ACFs in the $2 \mathrm{D}$ and $3 \mathrm{D}$ models. (Upper panel) The ACF as a function of $\delta R$ at fixed $\theta=45^{\circ}$ (by the red line) and the azimuthally averaged ACF (by the black line) obtained by the 3D simulations. (Lower panel) The same but for the 2D simulations.

This feature can also be captured from close inspection of the lower-left panel of Figure 4; that is, we see a number of clumps in the radial directions but they are not equidistant.

To compare the ACFs of the $2 \mathrm{D}$ and $3 \mathrm{D}$ results more quantitatively, we plot in Figure 6 the ACFs calculated based on the $3 \mathrm{D}$ simulations (upper panel) and those based on the 2D simulations (lower panel), respectively. In the upper panel the black line represents the azimuthally averaged ACF. As expected, from comparing the density contours shown in Figure 4 we see that the clump width is thinner in the $3 \mathrm{D}$ simulations than in the $2 \mathrm{D}$ simulation. In addition, we find that the $\mathrm{ACF}$ of the $3 \mathrm{D}$ simulations (red line in the upper panel) is similar to that of the 2D model (lower panel). However, the azimuthally averaged ACF is much smoother, indicating that the separation between the neighboring clumps is quite complicated, as we have mentioned above (see also Figure 5).

Finally, we plot the ACF as a function of $\delta \theta$ in Figure 7 based on the matter density contour data displayed in the lowerleft panel of Figure 4. The half-width of the clumps in the azimuthal direction is estimated at $\ell_{\mathrm{cl}}^{\theta} / 2 \sim 30 r_{\mathrm{S}}$, which is a factor of $\sim 2$ larger than that in the radial direction. This gives a qualitative difference in the widths of the clumps, depending on the direction.

We admit that the ACF analysis is of limited use, since we can easily understand from a quick look at the lower-left panel 


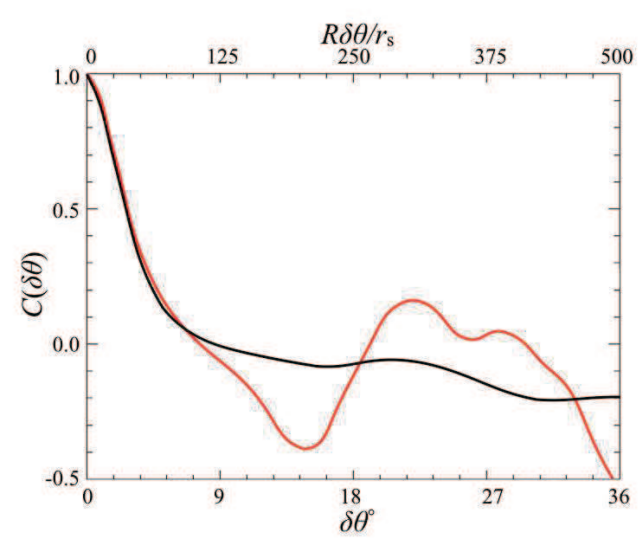

Fig. 7. As the upper panel of figure 6 , but for the ACF as a function of $\delta \theta$. We fix the value of $R=740 r_{\mathrm{S}}$.

of Figure 4 that each clump is stretched out nearly (but not precisely) in the azimuthal direction and that its length is much longer than $\sim 30 r_{\mathrm{S}}$, on the order of $R \sim$ several hundreds of $r_{\mathrm{S}}$. The reason why much a shorter correlation length is found in the ACF analysis at fixed $R$ resides in the fact that each clump does not have a straight shape but shows a wavy structure. This fact is essential when we discuss the obscuration of the central light by floating clumps (see the next section).

\section{Discussion}

\subsection{Formation Mechanism of Clumpy Outflows}

There are several physical mechanisms proposed for clump (or cloud) formation in gas flow; thermal instability (Field 1965), radiation-induced instability (Shaviv 2001), photo-bubble instability (Arons 1992), etc. When considering our particular case, we should keep in mind several key features confirmed by the 2D RHD simulations (see T13):

(1) clumpy structure appears in the layer where upward radiation force overcomes downward gravity force,

(2) a clump size is about one optical depth,

(3) there is an anti-correlation between the gas density and the absolute value of radiation force.

(4) temperature variations of some clumps are neither monotonic increase nor monotonic decrease.

Not all but some similar features are confirmed in the present 3D simulation. The first one is obvious; we are now considering radiation-pressure-driven outflow. The second feature can also be confirmed. The typical (radial) clump size and density are $\ell_{\mathrm{cl}}^{r} \sim 30 r_{\mathrm{S}} \sim 10^{8} \mathrm{~cm}$ and $\rho_{\mathrm{cl}} \sim 10^{-7} \mathrm{~g} \mathrm{~cm}^{-3}$, respectively (see the middle-left and lower-left panels of Figure 4). From these values we estimate the scattering optical depth $\left(\tau_{\mathrm{cl}}=\kappa_{\mathrm{es}} \rho_{\mathrm{cl}} \ell_{\mathrm{cl}}^{r}\right)$ to be a few. Note that optical depth is expected to be independent of the black hole mass, as long as the following scaling relations hold: $\rho_{\mathrm{cl}} \propto M_{\mathrm{BH}}^{-1}$ and $\ell_{\mathrm{cl}} \propto M_{\mathrm{BH}}$ (see T13). We do not have a clear correlation, however, between the matter density and radiation force, as was demonstrated by T13 (see their Figure 4). Although the precise origin of clump formation in the 3D simulations requires further investigation, we can at least say that the combination of the Rayleigh-Taylor instability in the radiation-pressure-supported atmosphere and a sort of radiation hydrodynamic instability seems to be involved with the clump formation.

The reason for asymmetric 3D shape of the clumps may be understood in terms of the anisotropic radiation field. That is, the radiation flux is highly super-Eddington in the radial direction, while it is not in the angular direction. Hence, the Rayleigh-Taylor instability grows to form a mushroom structure in the radial direction in the initial phase, followed by the formation phase of individual clumps (with a typical size of one optical depth) by a sort of radiation hydrodynamic instability (T14). Due to the differential rotation, however, such radially elongated clumps is soon stretched out in the angular direction, thus forming a torn sheet structure.

\subsection{Notes on the Numerical Resolution}

It is important to note that our 3D simulations produce larger clump sizes $\left(\sim 30 r_{\mathrm{S}}\right)$ than those obtained by the high-resolution 2D simulations ( $\sim 10 r_{\mathrm{S}}$, see T13). This is due partly to the $3 \mathrm{D}$ effects but poor resolutions in $3 \mathrm{D}$ simulations may also affect the clump size. In order to clarify this point, we performed $3 \mathrm{D}$ simulations with a grid spacing in the radial direction of $2.0 r_{\mathrm{S}}$ (half of that of the simulations presented in the manuscript), while keeping the same spacing in the azimuthal direction, for a shorter simulation time (10 s), finding that the averaged clump size is slightly less $\left(\sim 25 r_{\mathrm{S}}\right)$. We thus admit that the numerical resolution does affect the calculated clump size. Certainly, higher-resolution simulations are necessary to obtain a real clump size as future work.

\subsection{Comparison with Observation}

In this section we discuss the observational implications in terms of three quantities: variability timescale, ionization parameter, and volume filling factor.

\subsubsection{Variability timescale}

The clumpy nature of the outflow has been indicated thorough, e.g., X-ray spectral variations of luminous accretion flows, such as ULXs (e.g. Middleton et al. 2011; Pinto et al. 2016, 2017). When clumps pass across the observer's line of sight towards the central bright region, the observed luminosity is expected to be temporarily reduced, since the scattering optical depth of each clump is a few or so (see section 4.1). (The amount of reduction depends on the wavelengths of radiation, but we do not go into details in this discussion. The spectral variations are 
left as future work.) Along this line, let us estimate the variation timescales.

From the simulation results we find that clumps are seen at distances greater than

$R_{\mathrm{cl}} \sim 10^{3} r_{\mathrm{S}} \sim 10^{9.5}\left(\frac{M_{\mathrm{BH}}}{10 M_{\odot}}\right) \mathrm{cm}$,

and the clump length in the azimuthal direction is

$\ell_{\mathrm{cl}}^{\theta} \sim 10^{2} r_{\mathrm{S}} \sim 10^{8.5}\left(\frac{M_{\mathrm{BH}}}{10 M_{\odot}}\right) \mathrm{cm}$.

from the ACF analysis. It might be thought that the real clump lengths could be much longer, $\ell_{\mathrm{cl}}^{\theta} \sim 10^{3} r_{\mathrm{S}}$ at longest, from close inspection of the density contours (see subsection 3.2). But as a conservative estimate, we continue to use the value of $10^{2} r_{\mathrm{S}}$.

The clump velocities are, on the other hand, of the order of $\sim 0.3 V_{\mathrm{K}}$, where the Keplerian velocity is (see equation 12 )

$V_{\mathrm{K}}=\sqrt{\frac{G M_{\mathrm{BH}} R_{\mathrm{cl}}^{2}}{r_{\mathrm{cl}}^{3}}} \simeq 10^{8.6}\left(\frac{R_{\mathrm{cl}}}{10^{3} r_{\mathrm{S}}}\right)^{-1 / 2} \mathrm{~cm} \mathrm{~s}^{-1}$

where we set $r_{\mathrm{cl}} \simeq \sqrt{2} R_{\mathrm{cl}}$ (i.e., $\theta=45 \mathrm{deg}$ ). Then, the variation timescale is estimated to be

$t_{\mathrm{var}} \sim \frac{\ell_{\mathrm{cl}}^{\theta}}{0.3 V_{\mathrm{K}}} \sim 2.5\left(\frac{M_{\mathrm{BH}}}{10 M_{\odot}}\right)\left(\frac{R_{\mathrm{cl}}}{10^{3} r_{\mathrm{S}}}\right)^{1 / 2}\left(\frac{\ell_{\mathrm{cl}}^{\theta}}{10^{2} r_{\mathrm{S}}}\right) \mathrm{s}$,

In conclusion, the variability timescale could be several seconds for a $10 M_{\odot}$ black hole and could be even longer for a more massive black hole, since it is proportional to the black hole mass.

We also attempt to estimate the variation timescales directly from the simulation data by calculating the time variations of effective optical depth $\left(\tau_{\text {eff }}\right)$ by integrating the effective opacity ( $\sqrt{3 \kappa_{\mathrm{es}} \kappa_{\mathrm{ff}}}$ for $\left.\kappa_{\mathrm{es}} \gg \kappa_{\mathrm{ff}}\right)$ from the outer boundary of the simulation box to $r=300 r_{\mathrm{S}}$. We have chosen the radial distance of $300 r_{\mathrm{S}}$ since this layer crudely corresponds to the location of the mean photosphere in the sense that the effective optical depth is around unity. In Figure 8, we show the relative variations in the effective optical depth from the average value, postulating that optical depth variations will lead to time changes in the flux because of absorption by floating clouds above the mean photosphere. This is for the case that the observer is at infinite distance from the center in the directions of $\phi=30^{\circ}, 45^{\circ}$, and $60^{\circ}$ for a fixed $\theta=45^{\circ}$, where $\phi\left(\equiv \tan ^{-1} R / z\right)$ is the inclination angle.

Except during the first $3 \mathrm{~s}$, the initial transient phase, the optical depth varies on a timescale of several seconds. This would imply that the aperiodic fluctuation of the luminosity will be observed on this timescale. The amplitudes of variations would roughly be $\sim 50 \%-80 \%$ because of $\left|\Delta \tau_{\text {eff }}\right| \sim 0.2-0.3$, depending on the viewing angle. We admit, however, that this is a very crude estimation, and hence, we eventually need a 3D radiation transfer calculation to make the point clear.

This timescale is consistent with the variation timescale of a

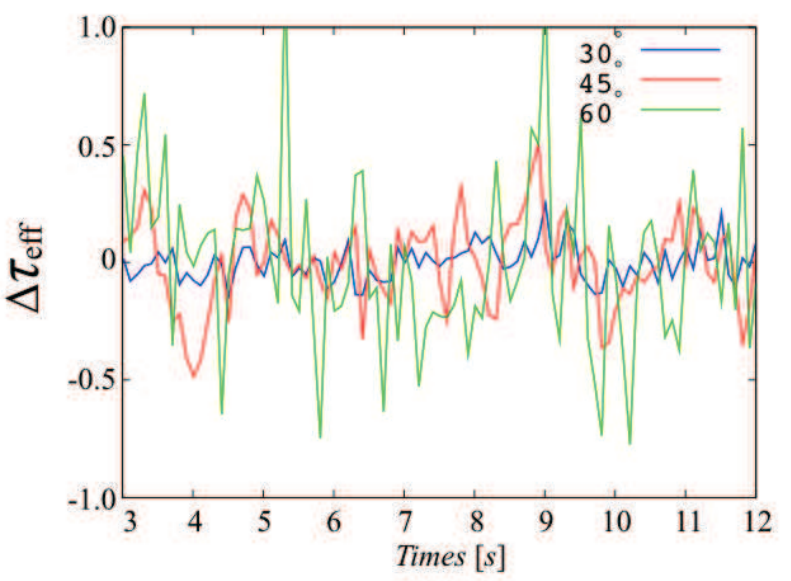

Fig. 8. Time variations of the effective optical depth with respect to the averaged one for a variety of the angles measured by a distant observer at $\phi=30^{\circ}, 45^{\circ}$, and $60^{\circ}$ for a fixed $\theta=45^{\circ}$.

ULX estimated by Middleton et al. (2011). That is, the mass of the central objects of the ULX is, at most, on the order of several tens of $M_{\odot}$. We can safely conclude that the central objects should be stellar-mass black holes.

Our result gives a significantly longer timescale than that estimated by $\mathrm{T} 13$ based on their $2 \mathrm{D}$ simulations. There are two main reasons for this discrepancy: (1) the typical length scale of each clump is systematically longer than the clump width from our $3 \mathrm{D}$ simulations, and (2) the rotation velocity turns out to be by some factor smaller than the local Keplerian value, although T13 estimated the timescale by assuming Keplerian rotation.

\subsubsection{Photoionization parameter and volume filling factor}

In order to discuss the observational properties of the clumpy outflow, we further calculate two key parameters characterizing the outflow clumps: the photoionization parameter and the volume filling factor (see T13).

Let us first estimate the photoionization parameter, which is defined as

$\xi=L_{\mathrm{x}} / n_{\mathrm{cl}} r^{2}$

where $L_{\mathrm{x}}$ is the luminosity in the $\mathrm{X}$-ray band and $n_{\mathrm{cl}}$ is the (average) gas number density of the clumps. The latter is related to the (average) optical depth for Thomson scattering of the clumps through

$\tau_{\mathrm{cl}}=n_{\mathrm{cl}} \sigma_{\mathrm{T}} \ell_{\mathrm{cl}}$

where $\sigma_{\mathrm{T}}$ is the Thomson cross-section, and $\ell_{\mathrm{cl}}$ is the average size of the clumps; i.e., $\ell_{\mathrm{cl}} \sim \sqrt{\ell_{\mathrm{cl}}^{r} \ell_{\mathrm{cl}}^{\theta}}$. Then, the photoionization parameter is estimated to be

$\xi \sim 10^{3} \tau_{\mathrm{cl}}^{-1}\left(\frac{L_{\mathrm{x}}}{0.1 L_{\mathrm{E}}}\right)\left(\frac{\ell_{\mathrm{cl}}}{30 r_{\mathrm{S}}}\right)\left(\frac{r}{10^{3} r_{\mathrm{S}}}\right)^{-2} \operatorname{erg~\mathrm {cm}\mathrm {s}^{-1}.}$

Equation (21) indicates that clumpy outflows are mildly ionized, 
as long as $\ell_{\mathrm{cl}} \sim 30 r_{\mathrm{S}}($ Kallman \& McCray 1982).

It is important to note that the ionization parameter does not explicitly depend on the black hole mass, although the X-ray luminosity, $L_{\mathrm{x}}$, weakly depends on it; $L_{\mathrm{x}} \sim L_{\mathrm{bol}}$ for Galactic sources (with $M_{\mathrm{BH}} \sim 10 M_{\odot}$ ) while $L_{\mathrm{x}} \sim 0.1 L_{\mathrm{bol}}$ for AGNs (with $M_{\mathrm{BH}} \sim 10^{8} M_{\odot}$ ). Here, $L_{\mathrm{bol}}$ is the bolometric luminosity. We assume $L_{\mathrm{bol}} \sim L_{\mathrm{E}}$ in the estimation given in equation (21). We thus expect line absorption features to be observed, in agreement with observations.

Let us next estimate the volume filling factor defined as

$\mathcal{F}=\frac{V_{\mathrm{cl}}}{4 \pi r_{\text {out }}^{3} / 3}$,

where $V_{\mathrm{cl}}\left(=M_{\mathrm{cl}} / n_{\mathrm{cl}} m_{\mathrm{p}}\right)$ is the volume occupied by the clumps, with $M_{\mathrm{cl}}$ being the total mass of the clumps, and $r_{\text {out }}$ is the size of the outflow region. Most of the mass of the outflow is contained in the clump, so that we find $M_{\mathrm{cl}} \sim$ $\dot{M}_{\text {out }}\left(r_{\text {out }} / v_{\text {wind }}\right)$, with $\dot{M}_{\text {out }}$ being the outflow rate and $v_{\text {wind }}$ being the radial wind velocity. Since $n_{\mathrm{cl}}$ is given by equation (20), we can obtain the filling factor as

$$
\begin{aligned}
\mathcal{F} \sim & 4.5 \times 10^{-3} \tau_{\mathrm{cl}}^{-1}\left(\frac{\dot{M}_{\text {out }}}{10 L_{\mathrm{E}} / c^{2}}\right)\left(\frac{v_{\text {wind }}}{0.1 c}\right)^{-1} \\
& \times\left(\frac{\ell_{\mathrm{cl}}}{30 r_{\mathrm{S}}}\right)\left(\frac{r_{\text {out }}}{10^{3} r_{\mathrm{S}}}\right)^{-2} .
\end{aligned}
$$

This is consistent with the filling factor estimated by T13, because the outflow rate, the wind velocity, and the clump size are similar to those in T13. The volume filling factor is independent of the black hole mass because $\dot{M}_{\text {out }} \propto M_{\mathrm{BH}}$, $\ell_{\mathrm{cl}}\left(\propto n_{\mathrm{cl}}^{-1}\right) \propto M_{\mathrm{BH}}$, and $r_{\text {out }} \propto M_{\mathrm{BH}}$. Thus, our results are applicable to AGNs, although we employ a black hole of 10 $M_{\odot}$ in the simulation. If we employ $r_{\text {out }} \sim 10^{5} r_{\mathrm{S}}$, the filling factor becomes around $4.5 \times 10^{-7}$, which is roughly consistent with observations of the BLR clouds of the AGNs at around $r \sim 1$ pc $\left(\sim 10^{5} r_{\mathrm{S}}\right.$ for $M_{\mathrm{BH}} \sim 10^{8} M_{\odot}$; Peterson 1997). More quantitative analyses are left as future work.

This work is partially supported by MEXT/JSPS KAKENHI Grant Numbers 15K05036, 16K05309, and 17H01102 (KO), 17K05383 (SM), and 17K14260 (HRT). Numerical computations were mainly carried out on a Cray XC30 at the Center for Computational Astrophysics, National Astronomical Observatory of Japan. This research was also supported by MEXT as a Priority Issue on Post-K computer (Elucidation of the Fundamental Laws and Evolution of the Universe) and JICFuS.

\section{References}

Arons, J. 1992, ApJ, 388, 561

Bachetti, M., Harrison, F. A., Walton, D. J., et al. 2014, Nature,514, 202 Boirin, L., Parmar, A. N., Barret, D., Paltani, S., \& Grindlay, J. E. 2004, A\&A, 418, 1061
Church, M. J., Reed, D., Dotani, T., Bałucińska-Church, M., \& Smale, A. P. 2005, MNRAS, 359, 1336

Davidson, K., \& Humphreys, R. M. 1997, ARA \& A, 35, 1

Di Stefano, R., \& Kong, A. K. H. 2003, ApJ, 592, 884

Done, C., Gierlinski, M., Kubota, A. 2007, A \& AR, 15, 1.

Eggum, G. E., Coroniti, F. V., \& Katz, J. I. 1988, ApJ, 330, 142

Elitzur, M. 2012, ApJL, 747, L33

Elitzur, M., \& Ho, L. C. 2009, ApJL, 701, L91

Fabbiano, G., Gioia, I. M., \& Trinchieri, G. 1989, ApJ, 347, 127

Fabrika, S. 2004, Astrophysics and Space Physics Reviews, 12, 1

Feng, H., \& Soria, R. 2011, NewAR, 55, 166

Field, G. B., 1965, ApJ, 142, 531

Fragile, P. C., Olejar, A., Anninos, P. 2014, ApJ, 796, 22

Fukue, J. 2004, PASJ, 56, 569

Fürst, F., Walton, D. J., Stern, D., et al. 2016. ApJ, 831, L14

Gladstone, J. C., Roberts, T. P., \& Done, C. 2009, MNRAS, 397, 1836

Gu, D., Sun, M.-Y., Lu, Y.-J., Yuan, F., Lin, J.-F. 2016, ApJ, 818, L4

Halpern, J. P. 1984, ApJ, 281, 90

Hashizume, K., Ohsuga, K., Kawashima, T., \& Tanaka, M. 2015, PASJ, 67,58

Inayoshi K., Haiman Z., Ostriker J. P., 2016, MNRAS, 459, 3738

Israel G. L., Belfiore, A., Stella, L., et al., 2017, MNRAS, 466, 48

Jiang, Y.-F., Stone, J. M., \& Davis, S. W. 2014, ApJ, 796, 106

Kaastra, J. S., Mewe, R., Liedahl, D. A., Komossa, S., \& Brinkman, A. C. 2000, A\&A, 354, L83

Kallman, T. R., \& McCray, R. 1982, ApJS, 50, 263

Kato, S., Fukue, J., \& Mineshige, S. 2008, Black-Hole Accretion Disks: Towards a New Paradigm (Kyoto: Kyoto Univ. Press)

Kawashima, T., Ohsuga, K., Mineshige, S., Heinzeller, D., Takabe, H., \& Matsumoto, R., 2009, PASJ, 61, 769

Kawashima, T., Ohsuga, K., Mineshige, S., Yoshida, T., Heinzeller, D., \& Matsumoto, R., 2012, ApJ, 752, 18

Kawashima,T.,Mineshige, S., Ohsuga, K., \& Ogawa, T. 2016, PASJ, 68, 83

King, A. 2003, ApJL, 596, L27

King, A. R., Davies, M. B., Ward, M. J., Fabbiano, G. \& Elvis, M., 2001, ApJ, 552L, 109

Kitaki, T., Mineshige, S., Ohsuga, K., Kawashima, T. 2017, PASJ, 69, in press

Kormendy, J., Ho, L. C. 2013, ARA\& A, 51, 511

Krumholz, M. R., \& Thompson, T. A. 2012, ApJ, 760, 155

Levermore, C. D. 1984, J. Quant. Spectrosc. Radiat. Transfer, 31, 149

Reynolds, C. S. 1997, MNRAS, 286, 513

Matsumoto, Y., Asahina, Y., Kudoh, Y., et al. 2016, arXiv:1611.01775

McKinney, J. C., Tchekhovskoy, A., Sạdowski, A., \& Narayan, R. 2014, MNRAS, 441, 3177

Middleton, M. J., Roberts, T. P., Done, C., \& Jackson, F. E. 2011, MNRAS, 411, 644

Miller, J. M., Fabian, A. C., Kaastra, J., Kallman, T., King, A. I., Proga, D., Raymond, J., Rreynolds, C. S. 2015, ApJ, 814, 87

Mineshige, S., Kawaguchi, T., Takeuchi, M., \& Hayashida, K. 2000, PASJ, 52, 499

Narayan, R., Sa̧dowski, A., \& Soria, R. 2017, MNRAS, 469, 2997

Narayan, R., Sạdowski, A., Soria R., 2017, MNRAS (arXiv:1702.01158)

Nicastro, F. 2000, ApJL, 530, L65

Nugis, T., \& Lamers, H. J. G. L. M. 2000, A \& A, 360, 227

Ogawa, T., Mineshige, S., Kawashima, T., Ohsuga, K., \& Hashizume, K. 2017, PASJ, 69, 33 
Ohsuga, K., \& Mineshige, S. 2011, ApJ, 736, 2

Ohsuga, K., Mori, M., Nakamoto, T. \& Mineshige, S, 2005, ApJ, 628, 368

Ohsuga, K., Mineshige, S., Mori, M., Kato, Y. 2009, PASJ, 61, 7

Okuda, T., \& Fujita, M. 2000, PASJ, 52, L5

Paczyńsky, B., \& Wiita, P. J. 1980, A \& Ap, 88, 23

Pakull, M. W., Soria, R., \& Motch, C. 2010, Nature, 466, 209

Peterson, B. M. 1997, An Introduction to Active Galactic Nuclei (New

York: Cambridge University Press)

Pinto, C., Middleton, M. J., Fabian, A. C. 2016, Nature, 533, 64.

Pinto, C., et al. 2017, MNRAS, 468, 2865.

Sạdowski, A., Narayan, R., McKinney, J. C., \& Tchekhovskoy, A. 2014, MNRAS, 439, 503

Shaviv, N. J. 2000, ApJ, 532, L137

Shaviv, N. J. 2001, ApJ, 549, 1093

Silk, J., Rees, M. J. 1998, MNRAS, 331, L1

Smith, N., Ganeshalingam, M., Chornock, R., et al. 2009, ApJL, 697, L49

Swartz, D., A., Ghosh, K. K., Tenant, A. F., Wu, K. 2004, ApJS, 154, 519.

Takahashi, H. R., \& Ohsuga, K. 2013, ApJ, 772, 127

Takahashi, H. R., Ohsuga, K., Kawashima, T., \& Sekiguchi, Y. 2016, ApJ, 826, 23

Takahashi, H. R., \& Ohsuga, K. 2017, ApJL, 845, L9

Takahashi, H. R., Mineshige, S., \& Ohsuga, K. 2017, arXiv:1712.02068

Takeuchi, S., Mineshige, S., \& Ohsuga, K. 2009, PASJ, 61, 783

Takeuchi, S., Ohsuga, K., Mineshige, S.,2013, PASJ, 65, 88 (T13)

Takeuchi, S., Ohsuga, K., Mineshige, S., 2014, PASJ, 66, 48 (T14)

Tombesi, F., Cappi, M., Reeves, J. N., \& Braito, V. 2012, MNRAS, 422,

L1

Tombesi, F., Cappi, M., Reeves, J. N., et al. 2013, MNRAS, 430, 1102

Turner, N. J., Blaes, O. M., Socrates, A., Begelman, M. C., \& Davis, S. W. 2005, ApJ, 624, 267

Ueda, Y., Yamaoka, K., \& Remillard, R. 2009, ApJ, 695, 888

Urquhart, R. \& Soria, R., 2016, MNRAS, 456, 1859

Vierdayanti, K., Mineshige, S., Ueda, Y. 2010, PASJ, 62, 239

Wang, J. M., Szuszkiewicz, E., Lu, F.-J., Zhou, Y.-Y. 1999, ApJ, 522, 839

Watarai, K.-y., Mizuno, T., \& Mineshige, S. 2001, ApJL, 549, L77

Watarai, K.-y., Mineshige, S. 2003, ApJ, 596, 421 